\title{
Aprendiendo mecatrónica mediante el diseño y construcción de una plataforma de lanzamiento de prototipos de cohetes de agua
}

\author{
Cristian Márquez, Rodrigo Agis, Antonio Cañas, Miguel Damas \\ Departamento de Arquitectura y Tecnología de Computadores \\ Universidad de Granada \\ Granada, España \\ crsmarqz@correo.ugr.es, (ragis, acanas,mdamas)@ugr.es
}

\begin{abstract}
Resumen. Este artículo describe un TFM del Máster en Ciencia de Datos e Ingeniería de Computadores de la Universidad de Granada, consistente en la implementación de una plataforma de lanzamiento de prototipos de cohetes de agua, donde se ha realizado un diseño mecatrónico que permite el control del sistema de forma remota desde el PC. El objetivo que se persigue con este trabajo es mostrar mediante un ejemplo práctico algunos de los conceptos y habilidades adquiridas en dicho Master relacionadas con la mecatrónica. El diseño parte del modelado 3D de cada pieza que interviene en el mecanismo, luego se seleccionan los dispositivos electrónicos que se utilizan para el control de la plataforma, y finalmente se desarrolla el sistema virtual que permite el control desde el PC. El mecanizado de piezas e integración de todos los sistemas se realizó en el laboratorio de robótica y mecatrónica del CITIC-UGR.
\end{abstract}

Palabras Clave: Mecatrónica, Plataforma de lanzamiento, modelado 3D, mecanizado, cohete de agua.

\begin{abstract}
This paper describes a TFM of the Master in Data Science and Computer Engineering at the University of Granada, which is implementing of a launching pad prototype rocket water, where there has been a mechatronic design that allows control system remotely from the PC. The objective sought to be achieved with this work is to show through a practical example some of the concepts and skills acquired in this Master related to mechatronics. The design of the 3D modeling of each piece involved in the mechanism, then the electronic devices used to control the platform, and finally the virtual system that allows control from the PC develops are selected. Machining parts and integration of all systems was conducted in the laboratory of robotics and mechatronics CITIC-UGR.
\end{abstract}

Keywords: Mechatronics, launch pad, 3D design, machining, water rocket. 


\section{Introducción}

Este proyecto se desarrolla en el marco de la iniciativa UGRASP (UGR Aero Space Program), impulsada por el Departamento de Arquitectura y Tecnología de Computadores de la Universidad de Granada para ayudar a los estudiantes a desarrollar sus habilidades de ingeniería (telecomunicaciones, mecánica, control, etc.) a través de misiones espaciales simuladas tales como vehículos voladores, robots móviles o plataformas de lanzamientos, entre otras.

Una plataforma de lanzamiento son las instalaciones y el área donde despegan los cohetes y las naves espaciales. Una base espacial (o lugar de despegue de cohetes) puede contener una o varias plataformas. Una plataforma de lanzamiento típica consiste básicamente en estructuras de servicio y líneas de abastecimiento [1].

En cuanto a las plataformas de lanzamientos de cohetes de agua, existen varios modelos y diseños, desarrollados por afícionados o grupos de estudiantes de escuelas secundarias que realizan proyectos de cohetes de agua (Fig. 1) [2].

Debido a esto, es escasa la información que se puede encontrar acerca de plataformas como las que se describe en este trabajo, donde se realizan tareas multidisciplinares en las que se conjugan varias ramas de la ingeniería, con el objetivo de lograr la integración de todos los elementos mecánicos y de control electrónico para un sistema de control y monitorización remoto. Entre los modelos que se encuentran en la web los más comunes son los de elaboración casera y accionamiento manual [3].

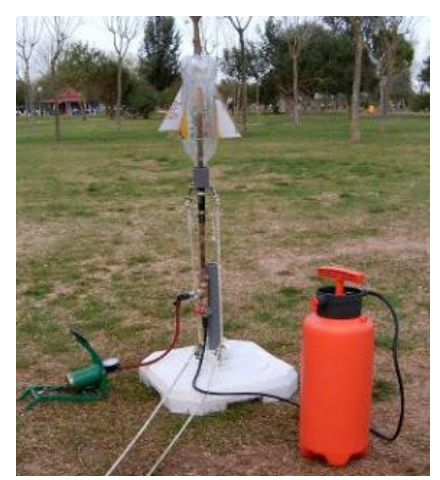

Figura 1. Plataforma de lanzamiento de cohetes de agua, de elaboración casera

Dentro de la propuesta de UGRASP, éste trabajo se plantea con el objeto de desarrollar capacidades de ingeniería multidisciplinar, aprendiendo de experiencias de grandes referentes en el área como la NASA y ESA, para motivar a los nuevos alumnos en su formación universitaria.

Para el desarrollo de la plataforma se ha realizado un diseño que simplifica la complejidad del control, incorporando elementos de hardware libre, actualmente con ilimitada información en la web sobre su uso y funcionamiento. En cuanto a la 
programación y control se ha diseñado una plataforma que permite la modificación o expansión del proyecto de forma fácil, donde con una base pequeña de conocimientos de programación se podría modificar parámetros de la plataforma.

El resto del artículo se divide en las siguientes secciones: en la sección 2 se hace una revisión del diseño mecatrónico del modelado de las piezas, la configuración de dispositivos electrónicos y el desarrollo del software que permite el control desde el ordenador. En la sección 3 se detalla el proceso de fabricación e implementación de la plataforma, desde el mecanizado de piezas hasta la integración de todos los sistemas. En la sección 4 se aportan los resultados obtenidos de esta experiencia, y por último en la sección 5 se presentan las conclusiones.

\section{Diseño del sistema mecatrónico}

El diseño del sistema incluye todo lo correspondiente a los esquemas desarrollados tanto en la parte mecánica así como también un pequeño diseño de la parte electrónica para el control.

Para el desarrollo del diseño mecánico se obtuvieron ideas y sugerencias del proyecto de la plataforma de lanzamiento de cohetes desarrollado en la asignatura de Mecatrónica y Sistemas Aeroespaciales del Máster de Ciencia de Datos e Ingeniería de Computadores, periodo 2014 - 2015. [6]

El diseño mecánico consta de dos partes, la primera determina toda la infraestructura donde se van a montar todas las piezas fijas y móviles además de los dispositivos que ayudan al control del proyecto, y la segunda parte es el sistema que permite el llenado y presurización del cohete (Fig. 2).

Para una visualización conceptual se desarrolló una vista en 3D (Fig. 3), la cual permite depurar y corregir de manera más detallada el diseño de la estructura previo al mecanizado de las piezas con la fresadora CNC del laboratorio.

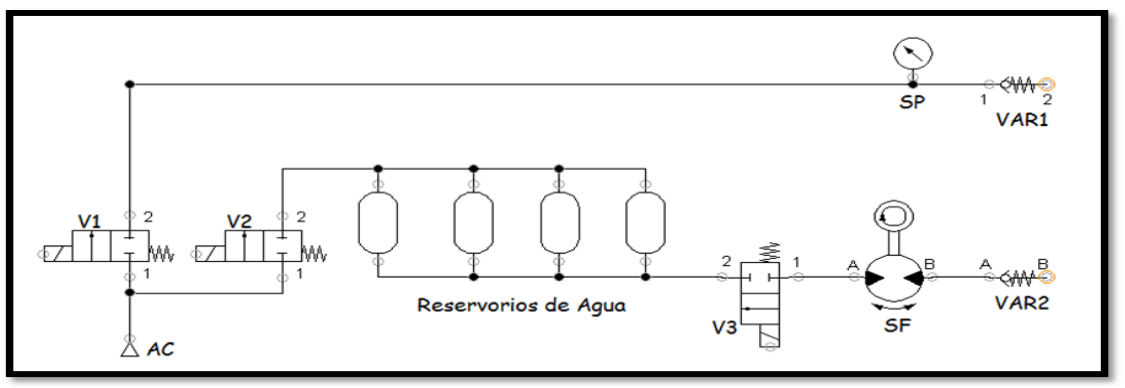

Figura 2. Diseño del sistema de llenado y presurizado del cohete

Donde:

V1, V2, V3: Válvulas Solenoides (12 Vdc).

$A C$ : Aire Comprimido (Compresor).

SP: Sensor de Presión de Aire.

$\boldsymbol{S F}$ : Sensor de Flujo de Agua.

VAR1, VAR2: Válvulas anti retorno. 
Para desarrollar las piezas definitivas se realizaron varios modelos previos en 3D, los mismos que permitieron pulir detalles para conseguir el diseño final, siguiendo un proceso de desarrollo y optimización.

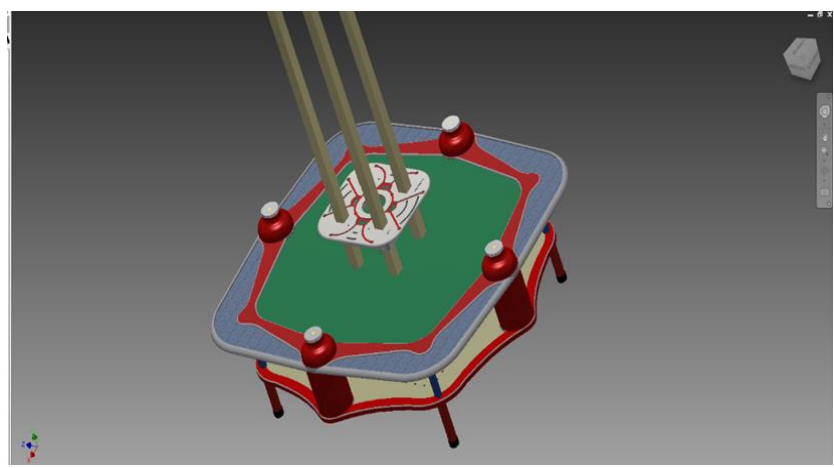

Figura 3. Diseño 3D de Ensamble de piezas

Para el diseño electrónico se pensó en varias soluciones para la elección de la plataforma de control, quedando como la más óptima la placa Arduino Uno. La comunidad Arduino ofrece muchas ventajas para el desarrollo de prototipos, además de disponer de una amplia gama de sensores e interfaces que permiten el manejo de periféricos de entrada y salida, digitales y analógicos.

Para una visualización previa al montaje en la placa de prototipo, se realizaron pruebas en el software de diseño Proteus 8 Profesional (Fig. 4).

Las pruebas realizadas con dicho software permiten simular los circuitos en condiciones ideales, sin tomar en cuenta la fuente de alimentación y la comunicación mediante bluetooth. Es importante resaltar estos detalles ya que durante la implementación e instalación se realizó una selección rigurosa que permitió un funcionamiento adecuado, muy similar al que se pudo observar durante las simulaciones.

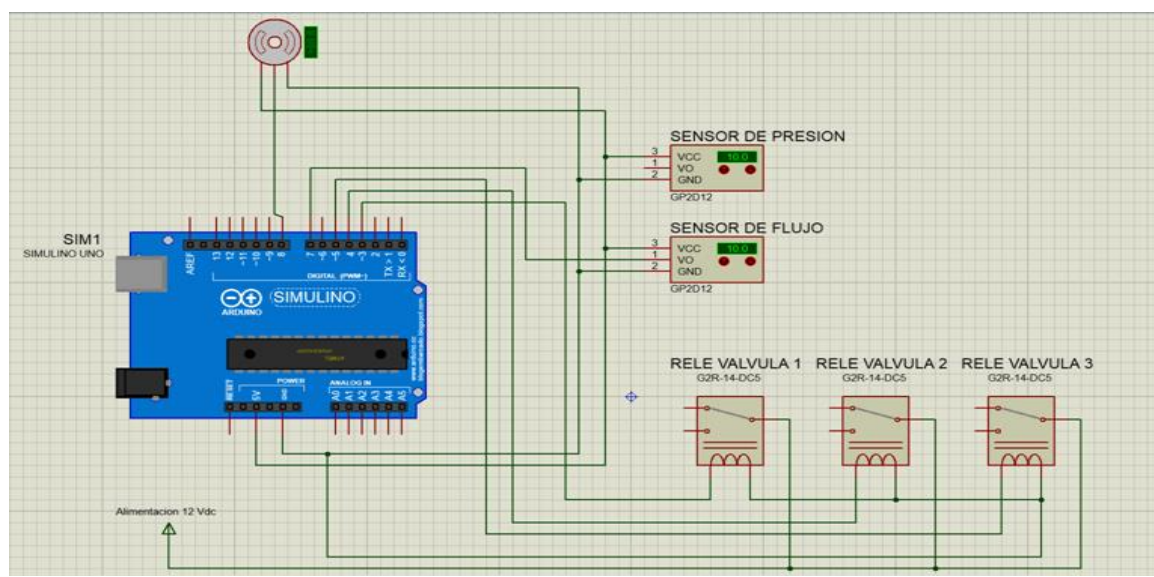

Figura 4: Esquema del circuito electrónico con placa de desarrollo Arduino 
Para el diseño del software partimos de que los dispositivos que se seleccionaron, en este caso la tarjeta Arduino, nos permiten comunicar con el software que queremos escoger, en este caso LabView. Para poder establecer una comunicación entre Arduino y LabView, se requieren de dos elementos muy importantes, uno es el ToolBox de Arduino para LabView que se puede encontrar en el package manager de LabView, y la segunda herramienta importante es el firmware que ha de cargarse en la placa Arduino, que permite comunicar dicha placa con el ToolBox previamente instalado en LabView [5].

Una vez desarrollado el circuito que permite el control, se estableció un esquema de funcionamiento representado mediante etapas y transiciones (Fig. 5), que sigue la secuencia de lanzamiento del cohete de agua. Es decir, que las entradas del circuito (sensores, pulsadores, temporizadores, etc.), a medida que vayan cumpliendo su función irán activando las salidas (válvulas, mecanismo de despegue) en concordancia con la secuencia de lanzamiento.

La secuencia comienza con el accionamiento del botón inicio desde el ordenador, para dar paso al accionamiento de la válvula de control de aire comprimido de todo el circuito, donde pasado un tiempo, se activa la válvula llenado de agua del cohete hasta que el sensor de caudal lo indique, para que luego se de paso a la etapa de presurización del cohete de la misma forma hasta que el sensor lo indique. A partir de la siguiente etapa se espera el pulso desde el ordenador, que permite accionar el mecanismo de despegue del cohete. Es importante mencionar que los estados de los actuadores y el valor de los sensores se pueden visualizar desde la plataforma de control en el ordenador.

\section{Fabricación de la plataforma de lanzamiento "SATURNIA"}

Para el proceso de fabricación de la plataforma de lanzamiento partimos del modelado y diseño de las piezas en AutoCAD [8], software que permite el desarrollo de planos de construcción en 2D (Fig. 6). Una vez que se han adecuado los planos de cada pieza, se procede con la exportación al software VCarve [10] que configura el programa de mecanizado de la Maquina $\mathrm{CNC}$, la cual corta, desgasta y taladra el material de forma automática en base al diseño cargado. El material que se seleccionó para la fabricación de las piezas fueron chapas de aluminio de 8 y $5 \mathrm{~mm}$ de espesor, tubos cuadrados de aluminio de $800 \mathrm{~mm}$ de largo, $50 \mathrm{~mm}$ de ancho y $2 \mathrm{~mm}$ de espesor.

Es importante mencionar que para el proceso de fabricación es necesario tener en consideración varios criterios del método constructivo para el mecanizado de piezas, además de algunos criterios de manejo y selección de las herramientas que permiten una adecuada sujeción de las piezas en construcción.

Mediante la configuración del software VCarve, el diseño se somete a un proceso de mecanizado virtual, donde se establece rutas que seguirá la máquina CNC (Fig. 7), para perforar, cajear (o desgastar parcialmente el material) y cortar. Es necesario saber el diámetro de las fresas que se disponen para establecer puntos de partida a conveniencia, con el fin de obtener las medidas deseadas. 


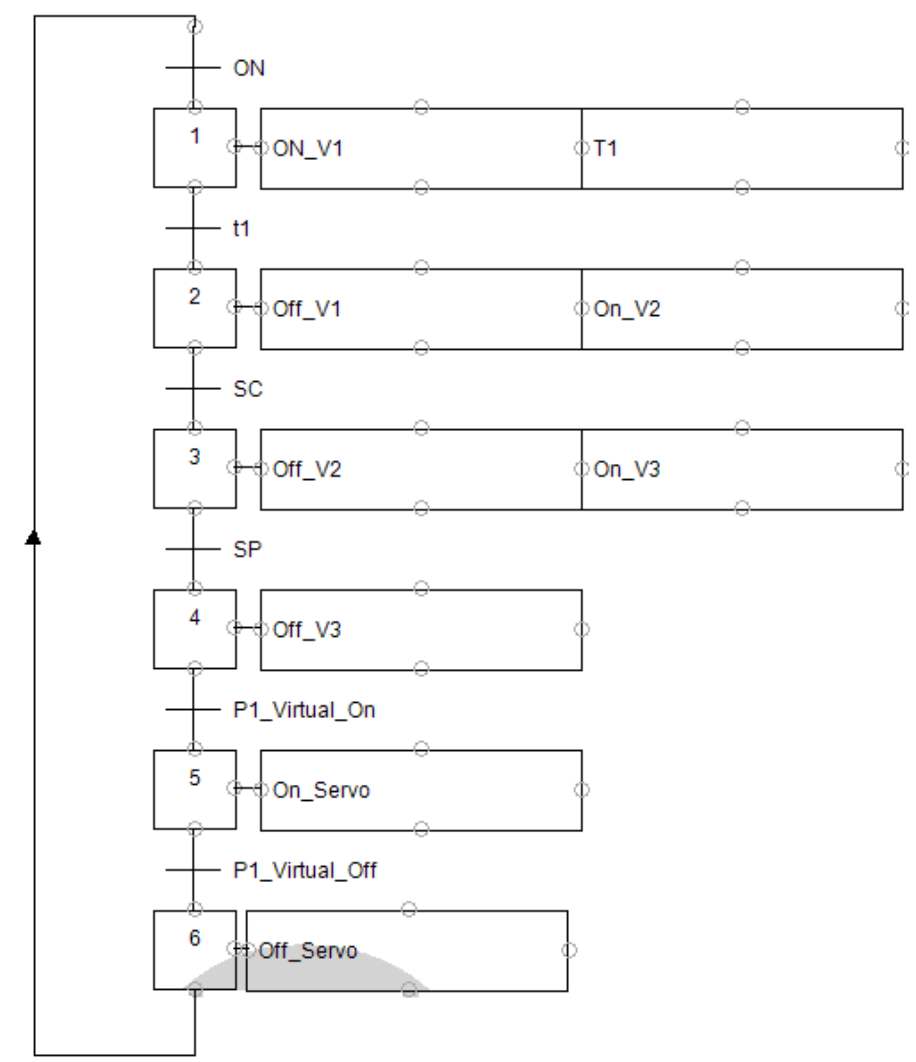

Figura 5. Diagrama de estados y transiciones de la plataforma de lanzamiento para prototipos de cohetes de agua

\section{Donde:}

V1, V2, V3: Válvulas de Solenoide 1, 2, 3

T1: Tiempo de transición 1

$S C$ : Sensor de Caudal

$\boldsymbol{S P}$ : Sensor de Presión

P1_Virtual: Pulso Virtual para activar o desactivar servomecanismo de lanzamiento

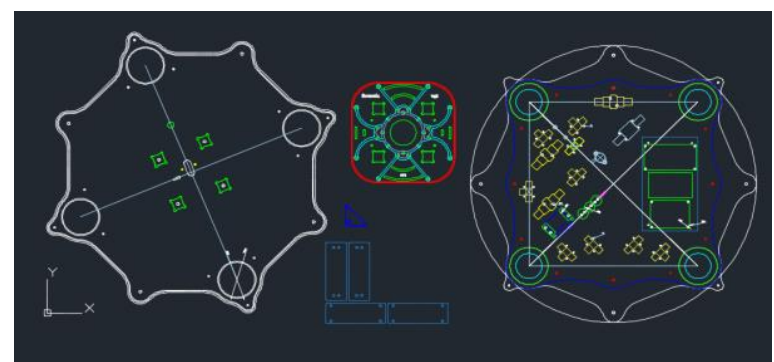

Figura 6. Diseño de piezas en 2D, en AutoCAD 


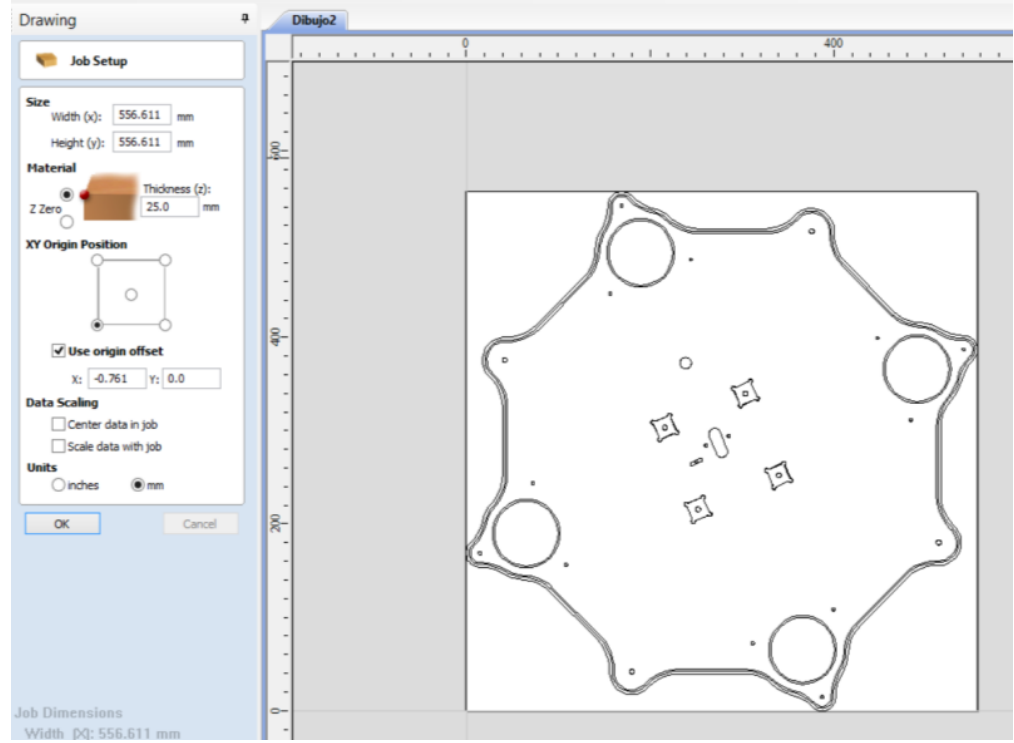

Figura 7. Proceso de cajeado y perforado en 2D previo al mecanizado mediante software VCarve.

Para el desarrollo de la etapa de control se seleccionaron los dispositivos electrónicos necesarios que deben intervenir en el control de la plataforma Saturnia, los mismos que fueron conectados de acuerdo al diseño simulado anteriormente (Fig. 8). Una vez implementado el circuito y con el firmware necesario se realizaron las primeras pruebas de funcionamiento del circuito, la lectura de sensores y la correcta activación de los actuadores.

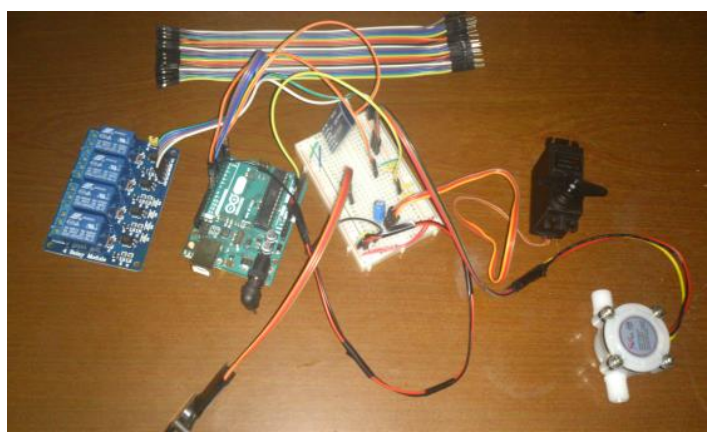

Figura 8. Circuito de control de la plataforma de lanzamiento de cohetes de agua, SATURNIA

Una vez realizada las pruebas de conexiones de la tarjeta Arduino se procedió con pruebas de conexión y transmisión de datos por bluetooth (Fig. 9), estableciendo comunicación entre el ordenador que alberga a LabView con la tarjeta Arduino, mediante la ToolBox de LabView para control de Arduino. 


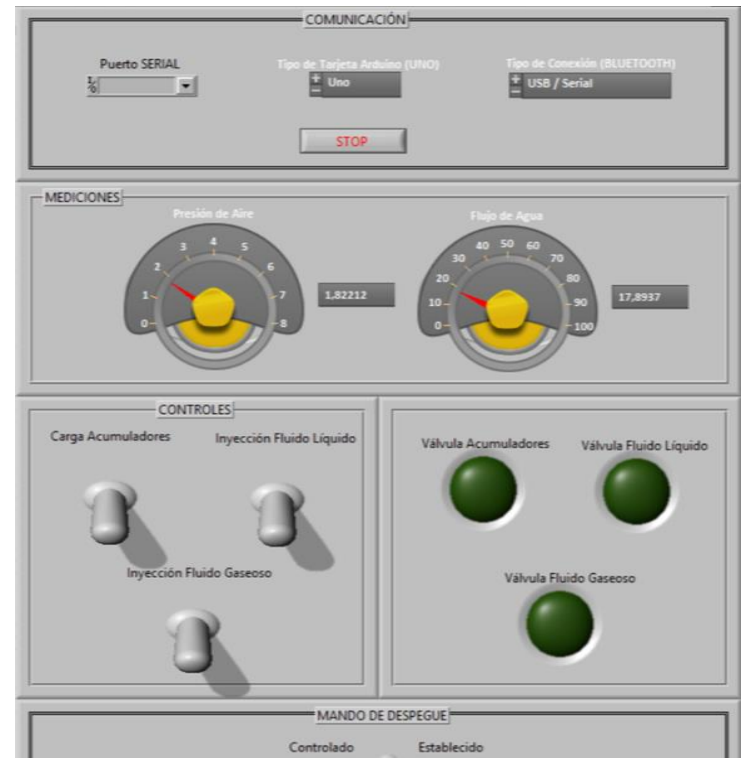

Figura 9. Panel de comunicación, control y visualización con LabView

La secuencia de funcionamiento se implementó en la plataforma LabView (Fig. 10), donde se realiza la comunicación bluetooth, la lectura de los sensores y el control de las salidas que intervienen en la plataforma de lanzamiento.

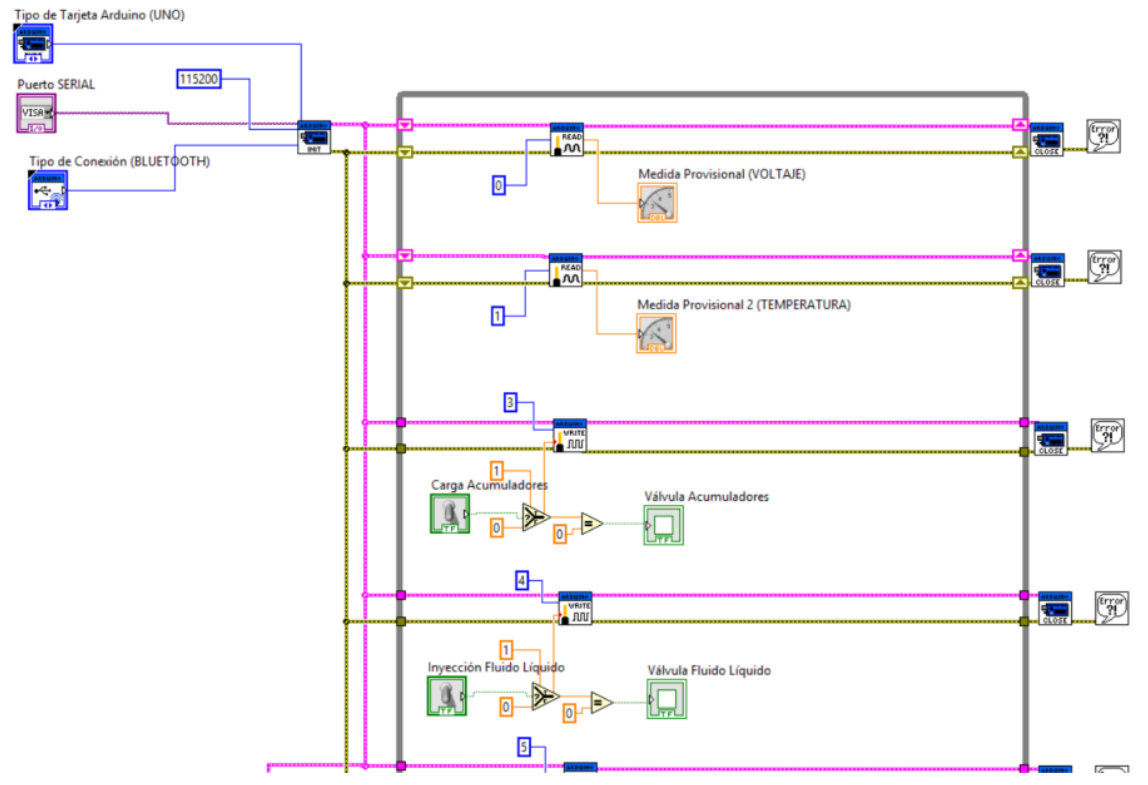

Figura 10. Programa de control y comunicación implementado en LabView 


\section{Resultados}

Una vez terminado con el armado y sujeción cuidadosa de las piezas fabricadas y de los dispositivos que fueron adquiridos para el control, se realizaron las pruebas de conexión eléctrica y neumática para la alimentación de los circuitos (Fig. 11).

Durante las pruebas de llenado se observaron pérdidas de agua en la conexione del cohete debido a la presión que generaba el aire comprimido, lo cual se pudo solucionar con una válvula anti-retorno.

Las lecturas de los sensores fueron calibradas utilizando el manómetro provisto en el compresor de aire y el volumen máximo que podía albergar cada cohete. Las válvulas anti-retorno que se fijaron a continuación de cada sensor permitieron obtener mejores medidas.

La comunicación bluetooth establecida entre Arduino y LabView permitió controlar la plataforma de lanzamiento en espacio abierto con un rango de distancia de hasta 20 metros aproximadamente.

La toma de conexión de los cohetes con la plataforma se diseñó de tal forma que pueda ser considerada de forma universal, es decir que los cohetes fabricados con cualquier tipo de botella de plástico puedan ser utilizados.

El mecanismo de despegue en principio tuvo unos pocos inconvenientes para permitir la liberación debido a la fricción entre los materiales, lo cual se solucionó realizando una pequeña modificación en la sujeción del mecanismo de accionamiento.

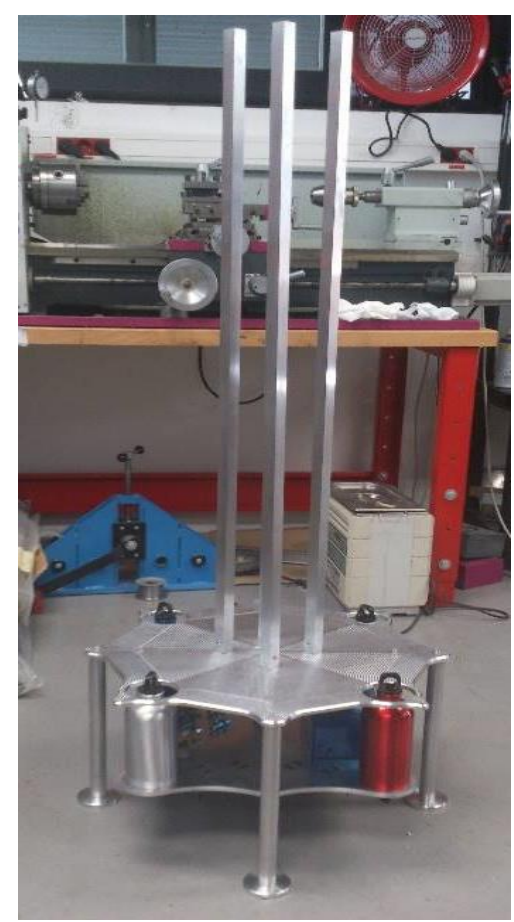

Figura 11. Plataforma ensamblada 


\section{Conclusiones}

En éste trabajo se ha mostrado el desarrollo de un proyecto para la construcción de una plataforma de lanzamiento de prototipos de cohetes de agua, para lo que se ha tenido que recurrir a varias disciplinas de la ingeniería, es decir a la mecatrónica, para por ejemplo, la construcción mecánica y el diseño de la electrónica de control, o el desarrollo de una aplicación para su monitorización de forma remota.

Durante el diseño y la construcción de la plataforma de lanzamiento para cohetes de agua fue necesario contar con un adecuado esquipo de herramientas virtuales y físicas que permitieron la fabricación de las piezas.

Durante las pruebas de funcionamiento de la plataforma se comprobó que el diseño de llenado y presurización del cohete fue el más óptimo ya que permitió un adecuado despegue del cohete.

El control de la plataforma por medio de una comunicación bluetooth ha permitido la conexión instantánea del ordenador con la plataforma tanto para leer como para controlar los dispositivos conectados.

La integración de varios sistemas requiere de una adecuada planificación que parte de un tiempo adecuado para el diseño y la fabricación, y luego la implementación y las pruebas que se realicen.

Finalmente señalar que debido al presupuesto limitado varias ideas se han quedado fuera, pero se espera que en un futuro se pueda contar con algo más de presupuesto para las mejoras del proyecto Saturnia, tales como la implementación del control para sistemas móviles o añadir un mecanismo de lanzamiento angular para dar trayectorias curvas al cohete, entre otras.

\section{Referencias}

1. Water Rocket Simulator. http://www.grc.nasa.gov/WWW/K12/rocket/BottleRocket/sim.htm

2. Experciencia, http://www.experciencia.com/festa-de-la-ciencia-en-barcelona/

3. Cohetes Propulsados por agua, Artusa, Juan Ignacio. http://www.astroeduc.com.ar/ COHETES\%20PROPULSADOS\%20POR\%20AGUA\%20ISFN.pdf

4. Rocket Activity Water Rocket Launcher. http://www.nasa.gov/pdf/ 153405main_Rockets Water_Rocket_Launcher.pdf

5. VI Package Manager http://jki.net/vipm

6. Goddard, Robert (2002), Rockets, New York: Dover Publications, ISBN 978-0-486-42537-5

7. Cohetes de agua: C. J. Gommes, A more thorough analysis of water rockets: moist adiabats, transient flows, and inertials forces in a soda bottle. https://es.wikipedia.org/wiki/ Cohete_de_agua

8. Autocad. http://www.autodesk.es/products/autocad/overview (Autocad)

9. Inventor. http://www.autodesk.es/products/inventor/overview (Inventor)

10. Vcarve. http://www.vectric.com/products/vcarve.htm

11. Labview. http://www.ni.com/labview/esa/

12. FluidSIM. http://www.fluidsim.de/fluidsim/index5_e.htm 